J. Fish. Sci. Tech. 5(2), 97 102, 2002

\title{
Fungicidal Activity of Substance Purified from Marine Fungus Metabolites against Pyricularia oryzae
}

\author{
Hee-Guk Byun and Se-Kwon Kim* \\ Department of Chemistry, Pukyong National University, \\ Busan 608-737, Korea
}

(Received January 2002, Accepted May 2002)

\begin{abstract}
Pyricularia oryzae (P. oryzae), the cause of rice blast, is one of the most important fungal pathogens of rice. Seventy strains of marine fungi were isolated from marine algae, and it was measured antifungal activity against $P$. oryzae. Metabolites of marine fungus A-248 wh ch isolated from marine algae showed strong antifungal activity against $P$. oryzae. The antifungal substance from the metabolites of marine fungus $\mathrm{A}-248$ was extracted with ethylacetate, and then purified by preparative TLC and reverse-phase HPLC. The minimum inhibitory concentration (MIC) value was $0.18 \mu \mathrm{g} / \mathrm{mL}$ for the antifungal activity of the sut stance purified from A-248 metabolites. The purified substance was similar to antifungal act vity of rhizoxin, which is a commercial antifungal agent.
\end{abstract}

Ke:' words: Marine algae, Marine fungus, Pyricularia oryzae, Antifungal substances

\section{Introduction}

Pyricularia oryzae ( $P$. oryzae), which is responsible for causing blast in rice, is one of the major pathogens in rice culture because of its widespread occurrence and destructive nature (Manandhar et al., 1998a). The pathogenic fungus directly penetrates into the rice plant from a cellular structure called an uppressorium that is formed at the tip of the germ $t a b e$. And the fungus can attack any aerial part of the rice plant, including seeds, in which the fungus may overwinter for several years (Manandhar et al., 1998b). A continuous effort has been made to control this disease, especially by using fungicides (Bell and Wheeler, 1986) and breeding cultivars resistant to the disease (Bonman et al., 1992). However, these fungicides are adversely effect on the quality of the rice production, environment, and breecing of rice. The consecutive use also brings abcut a problem due to the differentiation of $P$. oryzae races, which cause the emergence of new mutants.

*Corresponding author: sknkim@ mail.pknu.ac.kr
In recently, pollution problems in the environment and the toxic effects of synthetic chemicals on non-target organisms have prompted investigations on exploiting pesticides of plant origin. Therefore, an environment-friendly method with biocontrol agents is recognized as an alternative approach to control this disease (Kim et al., 1997; Lange et al., 1993). The use of natural fungicides and biological control by antagonistic microorganisms are a plausible ways to take this approach (Yeo et al., 1994). The fungicides such as taxol, vinblastin, griseofulvin, rhizoxin and flavonoids showed the characteristic curling effect on mycelia of rice plant pathogenic fungus, $P$. oryzae, germinated in yeast extract solution (Kobayashi et al., 1996; Padmavati et al., 1997). Eventhough several studies have reported the isolation of induced resistance in rice under laboratory conditions, demonstrations in the field are very limited.

In this study, marine fungi producing antifungal substances were screened and isolated from marine algae. Antifungal substance against $P$. oryzae was purified from the metabolites of marine fungus, and was elucidated morphological characterization of $P$. oryzae by purified compound. 


\section{Materials and Methods}

\section{Preparation of conidia suspension}

$P$. oryzae was grown on a slant culture medium consisted of yeast extract $0.2 \%$, soluble starch $1 \%$ and agar $2 \%$ at $27^{\circ} \mathrm{C}$. The conidia were collected on 12 to 14 days after inoculation by suspending in sterilized water. The conidia suspension was filtered to separate from mycelia. The filtrate was added a $2 \%$ solution of yeast extract and adjusted to the concentration of $0.02 \%$. The aliquot of the conidia suspension was observed under the microscope to count the number of conidia before adding yeast extract. The suspension was adjusted to $4 \times 10^{4}$ con$\mathrm{idia} / \mathrm{mL}$ by adding sterilized water.

\section{Isolation of fungi from marine algae}

Marine algae was washed twice with each $2 \mathrm{~mL}$ of sterilized $50 \%$ seawater containing chloramphenicol in a sterilized test tube, and then steeped in the same solution $(4 \mathrm{~mL})$ for $24 \mathrm{~h}$ in a sterilized plate. The plate was incubated at $20^{\circ} \mathrm{C}$ for 7 days, and mycelia were taken to an agar plate (glucose $2 \%$, polypeptone $1 \%$, yeast extract $0.5 \%$, chloramphenicol $200 \mu \mathrm{g} / \mathrm{mL}$, penicillin $\mathrm{G} 50 \mu \mathrm{g} / \mathrm{mL}$ and agar $2 \%$ in $90 \%$ seawater, $\mathrm{pH} 6.5$ ). The agar plate was incubated at $20^{\circ} \mathrm{C}$, and the mycelia grown to the edge of plate was taken to a plate of $1 / 2$ potato dextrose (PD) agar (a half nutrient, 50\% seawater).

The seed culture of marine fungi was preformed by inoculating mycelia fragments of strain A-248 into $100 \mathrm{~mL}$-Erlenmeyer flasks containing $30 \mathrm{~mL}$ of $1 / 2 \mathrm{PD}$ medium at $20^{\circ} \mathrm{C}$ for 3 days. Five milliters of this seed culture transferred into each 1 L-Erlenmeyer flasks containing $300 \mathrm{~mL}$ of $1 / 2 \mathrm{PD}$ medium (total $3 \mathrm{~L}$ ) at $20^{\circ} \mathrm{C}$ for 21 days.

\section{Measurement of antifungal activity}

A 96-well plate was used for the bioassay. The first, second and last columns were preserved for positive and negative controls. Rhizoxin was used for positive control with the final concentrations of $5.00 \mu \mathrm{g} / \mathrm{mL}, 1.67 \mu \mathrm{g} / \mathrm{mL}, 0.56 \mu \mathrm{g} / \mathrm{mL}, 0.19 \mu \mathrm{g} / \mathrm{mL}, 0.062$ $\mu \mathrm{g} / \mathrm{mL}, 0.021 \mu \mathrm{g} / \mathrm{mL}, 6.86 \mathrm{ng} / \mathrm{mL}$ and $2.29 \mathrm{ng} / \mathrm{mL}$. One column of 96-well plate was used for one test material with eight different concentrations. The assay plates were incubated at $27^{\circ} \mathrm{C}$ for $16 \mathrm{~h}$, and the shape of mycelia germinated from conidia was obser- ved and compared with controls under an inverted Microscope (TMS, Nikon Co., Tokyo, Japan).

The indices of hyphal growth inhibition used for quantitative estimations were divided into five classes $(-, \pm,+,++$, and +++$)$ according to the length of mycelia germinated from conidia by Kobayashi et al. (1996) method.

\section{Purification of antifungal substances}

The culture broth ( $3 \mathrm{~L})$ was added $80 \%(\mathrm{v} / \mathrm{v})$ acetone $3 \mathrm{~L}$, and then shaken by magnetic stirrer for $1 \mathrm{~h}$ at room temperature as shown in Fig. 1. The mixture solution was centrifuged $(2,000 \times \mathrm{g}, 15 \mathrm{~min})$ at $4^{\circ} \mathrm{C}$, and then the supernatant was concentrate by an evaporator. The resulting aqueous solution was extracted three times each $3 \mathrm{~L}$ of ethyl acetate. The ethyl acetate extract was dried over $\mathrm{N}_{2}$ gas and concentrated in vacuum dryer.

Ethyl acetate extract was further purified using preparative TLC (PTLC) glass plates $(0.5 \mathrm{~mm}$ silica gel $60 \mathrm{~F}_{254}$, Merck, Darmstadt, Germany). After development in chloroform-methanol (20:1), the

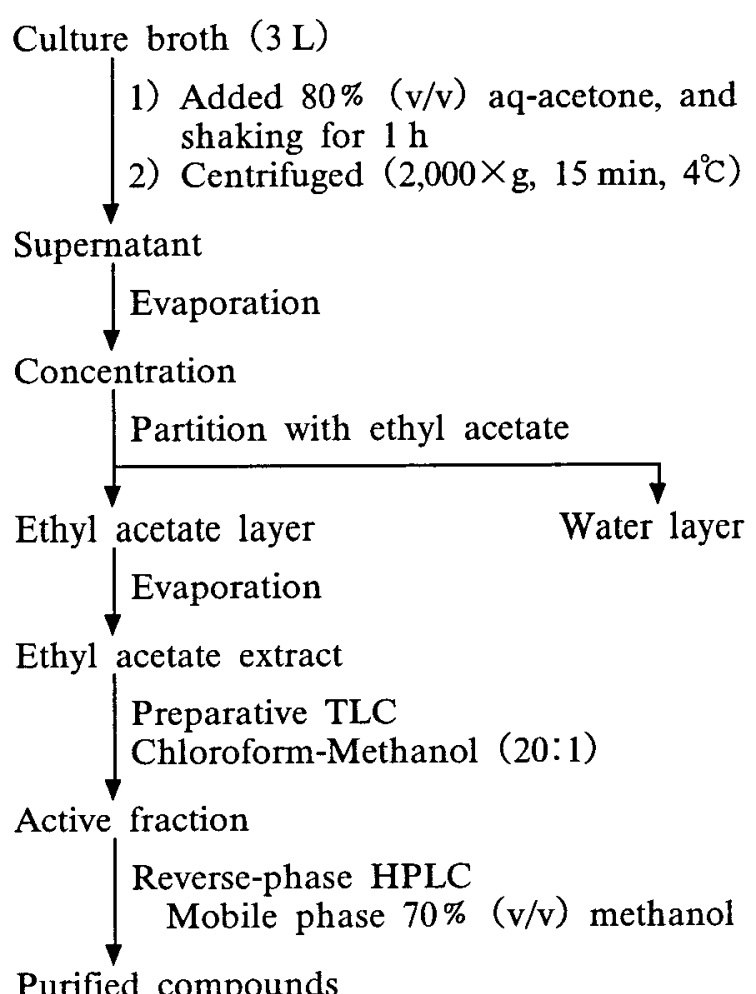

Purified compounds

Fig. 1. Isolation and purification scheme of antifungal substances from marine fungus metabolites. 
PTLC plate was divided into fourteen sections from top to bettom, which were scraped off the plates. The fracions were eluted from the silica with chloroform-methanol $(1: 1)$, and the eluates were recovered by centrifuging $(850 \times \mathrm{g}, 15 \mathrm{~min})$. After evaporation in vacuum at $40^{\circ} \mathrm{C}$, the fractions were dissolved in methanol, and tested for antifungal activity u.ing a 96-well microplate bioassay.

The aclive fraction obtained from the PTLC was further p.rified by HPLC (Shimizu Co., Tokyo, Japan) using a pre-packed ODS $\mathrm{C}_{18}$ column (Develosil OD $-\mathrm{HG}^{-} 5, \phi 20 \times 250 \mathrm{~mm}$, Nomura Chemical Co., LTD, Japan). The column was developed at a flow rate of $9 \mathrm{~mL} / \mathrm{min}$ by a step-wise of $70 \%$ methanol. Antif.ngal activity of individual peak was measured. The active fractions were collected and concentrated by the evaporator, and then were purified with meth.anol in the same column.

\section{Results and Discussion}

\section{Screening and culture of marine fungus}

Seventy strains of marine fungus were isolated from marine algae, and were measured antifungal activity against $P$. oryzae. In the results, marine fungus A-248 showed a strong antifungal activity against $P$ oryzae. The antifungal activity of marine fungus $m$ tabolites against $P$. oryzae was measured by using mycelia shape of $P$. oryzae under an op- tical microscope. Rhizoxin caused the characteristic curling and swelling effect on mycelia as shown in Fig. 2. Morphological deformations observed on mycelia of fungi induced by synthetic agents have been known (Richmond, 1975) and were applied for screening of bioactive metabolites (Gunji et al., 1983; Beppu, 1993; Sakamoto et al., 1995). The screening detected morphological changes of fungal mycelia grown by $50 \mu \mathrm{L}$ cultured broth in 96 well plates. Comparison of antifungal activity of culture broths of various marine fungi against $P$. oryzae would be made at the same dilution, which are usually at the same row of 96-well plate.

Marine fungus A-248 showed strong antifungal activity on pathogenic fungus, $P$. oryzae. A petridish plate of the strain fungus A-248 grown on $1 / 2$ PD agar was used to inoculate $100 \mathrm{~mL}$ Erlenmeyer flasks containing $30 \mathrm{~mL}$ of $1 / 2 \mathrm{PD}$ broth. The broth was cultured for 3 days at $20^{\circ} \mathrm{C}$. Five milliters of the seed pre-culture were transferred into $300 \mathrm{~mL}$ of the $1 / 2 \mathrm{PD}$ broth (total $3 \mathrm{~L}$ ) in a $1 \mathrm{~L}$ flask. The culture was carried out at $20^{\circ} \mathrm{C}$ for 21 days under stationary condition. The marine fungus A-248 isolated from marine algae produced the antifungal substance at $20^{\circ} \mathrm{C}$, and $\mathrm{pH} 8.0$.

Purification and extraction of antifungal substance

The antifungal substances from culture broth (3 L) of marine fungus A-248 was extracted three
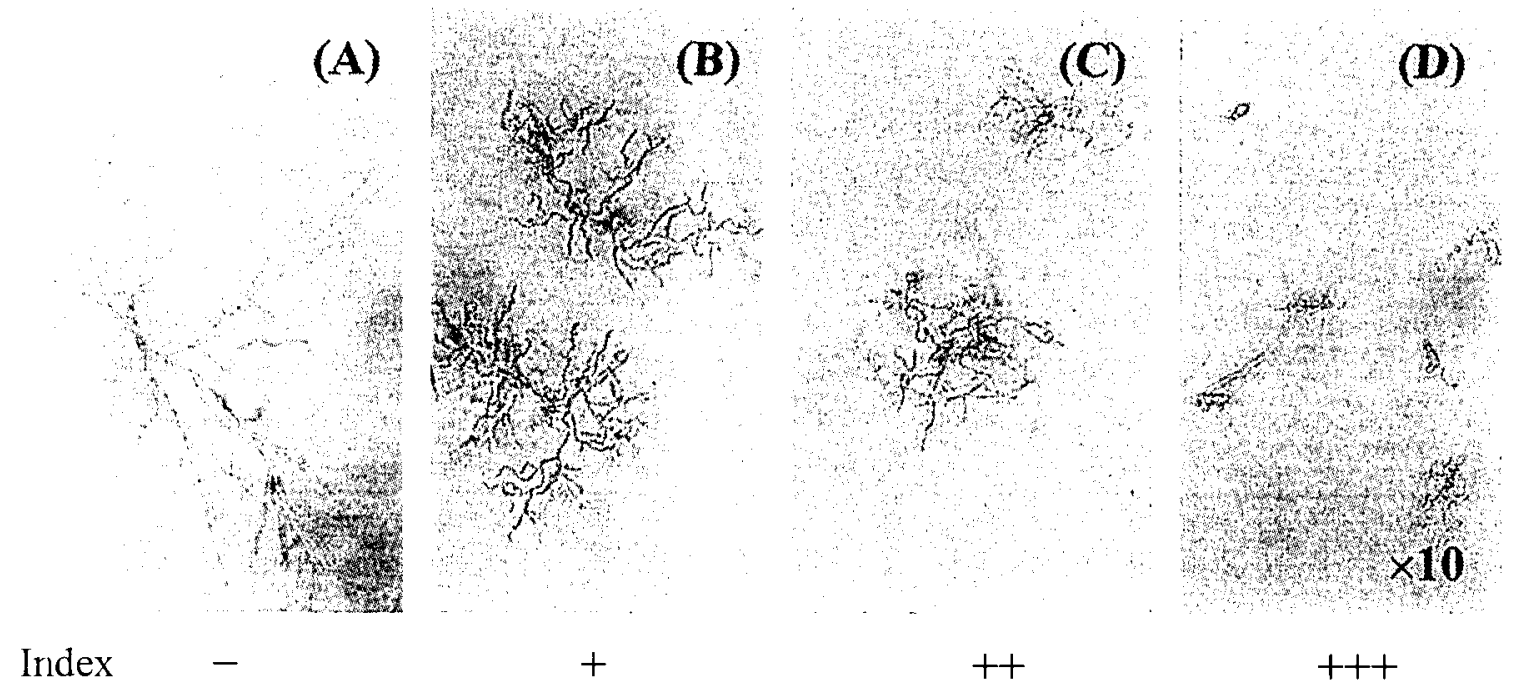

Fig. 2. Index of antifungal activity against $P$. oryzae induced by rhizoxin. Conidia were incubated in sterilized water containing $0.02 \%$ yeast extract with or without rhizoxin. 
Table 1. Antifungal activity against $P$. oryzae of fractions isolated by PTLC

\begin{tabular}{ccccccccccc}
\hline $\begin{array}{c}\text { PTLC } \\
\text { fraction }\end{array}$ & $\begin{array}{c}\mathrm{Rf} \\
\text { value }\end{array}$ & $\begin{array}{c}\text { Yield } \\
(\mathrm{mg})\end{array}$ & 400.00 & 133.33 & 44.44 & 14.81 & 4.94 & 1.65 & 0.55 & 0.18 \\
\hline 1 & 0.93 & 0.3 & $\pm \pm^{2}$ & - & - & - & - & - & - & - \\
2 & 0.88 & 0.7 & +++ & + & + & - & - & - & - & - \\
3 & 0.83 & 1.1 & +++ & +++ & +++ & + & \pm & - & - & - \\
4 & 0.76 & 21.0 & + & \pm & - & - & - & - & - & - \\
5 & 0.65 & 7.1 & +++ & \pm & - & - & - & - & - & - \\
6 & 0.57 & 3.9 & +++ & ++ & + & \pm & - & - & - & - \\
7 & 0.52 & 2.3 & +++ & +++ & ++ & \pm & - & - & - & - \\
8 & 0.47 & 1.2 & +++ & ++ & + & \pm & - & - & - & - \\
9 & 0.40 & 1.2 & +++ & +++ & +++ & +++ & ++ & + & \pm & - \\
10 & 0.33 & 0.4 & +++ & +++ & +++ & ++ & + & \pm & - & - \\
11 & 0.30 & 1.1 & +++ & +++ & +++ & + & \pm & - & - & - \\
12 & 0.20 & 0.5 & + & + & \pm & - & - & - & - & - \\
13 & 0.11 & 0.3 & + & \pm & - & - & - & - & - & - \\
14 & 0.00 & 5.5 & + & \pm & - & - & - & - & - & - \\
\hline
\end{tabular}

${ }^{12} \mathrm{MIC}$, minimum inhibitory concentration.

2),,,,++++++ \pm- ; levels were estimated by Kobayashi et al. (1996) method.

times with each $3 \mathrm{~L}$ of ethyl acetate, and the yield was $51 \mathrm{mg}$. The PTLC chromotogram was developed with chloroform-methanol (20:1) using the ethylacetate extract. The PTLC fractions were divided into fourteen sections from top to bottom (fraction 1 to 14). Fractionates were dissolved in methanol and tested for antifungal activity using a 96-well microplate bioassay. Among the antifungal activity of each PTLC fraction against $P$. oryzae, fraction 9 (Rf, 0.40) was the highest at minimum inhibitory concentration (MIC) value $1.65 \mu \mathrm{g} / \mathrm{mL}$ (Table 1), and yield was $1.2 \mathrm{mg}$. The shapes observed under the microscope were curling, swelling and bead shape indicated the deformation of mycelia.

The active fraction 9 obtained using PTLC was fractionated by HPLC using a pre-packed ODS column (Fig. 3). Antifungal activity against $P$. oryzae of the each fraction was compared with rhizoxin, which is the commercial antifungal agent. The antifungal agent, rhizoxin, was showed the characteristic curling and swelling effect at the concentration of $0.19 \mu \mathrm{g} / \mathrm{mL}$. The antifungal activity of active fraction purified from marine fungus A-248 by HPLC was $0.18 \mu \mathrm{g} / \mathrm{mL}$ as MIC value, and yield was $0.8 \mathrm{mg}$. The compound was strongly inhibited the hyphal growth by curling and swelling effect at lower concentration (Fig. 4). Inoue et al. (1995) reported that sonchifolin extracted from leaf extracts of Yacon (Smallanthus sonchifolius) exhibits the highest fungicidal activity ( $\mathrm{ED}_{50}$ value, $22 \mathrm{ppm}$ ) against $P$. oryzae. Suzuki et al. (1996) reported that 5-(8'Z- heptadecenyl) antifungal activity of resorcinol isolated from etiolated rice seedlings was about $40 \mu \mathrm{g} /$ $\mathrm{mL}$ as $\mathrm{ED}_{50}$ value. Naringenin and kaempferol show a significant inhibition of spore germination of $P$. oryzae at $7 \mu \mathrm{g}$ (Padmavati et al., 1997). Earlier studies demonstrated that while naringenin and sakuranetin inhibit spore germination of $P$. oryzae at 25 ppm and 5 ppm, respectively (Kodama et al., 1992). Bae et al. (2000) reported that antifungal protein having antifungal activity $(5.9 \mu \mathrm{g} / \mathrm{mL})$ against $P$. oryzae was isolated from Paenibacillus macerans PM1. Antifungal activity of compound purified

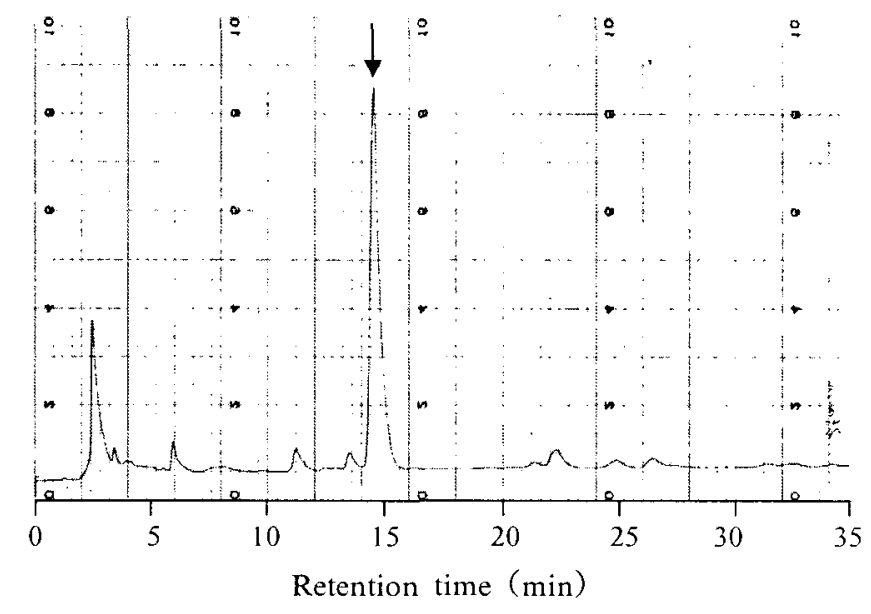

Fig. 3. Separation of the antifungal substances from active fraction 9 of PTLC by HPLC. The separation was performed with stepwise $(70 \%$ methanol, $35 \mathrm{~min})$ at a flow rate of $9 \mathrm{~mL} / \mathrm{min}$. Elution was monitored at $210 \mathrm{~nm}$. 


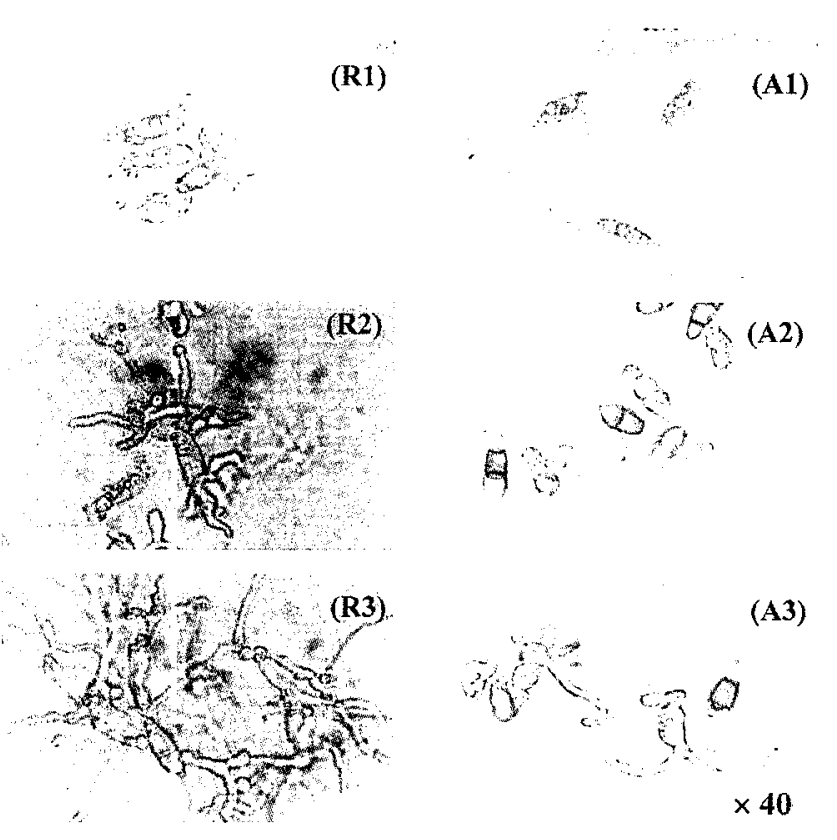

Fig. 4. Comparison of curling and swelling effect of mycelia germinated from conidia of $P$. oryzae induced by rhizoxin and anti ungal substance purified from marine fingus $\mathrm{A}^{-2} 248$ metabolite.

Rhizoxin (R1), $1.67 \mu \mathrm{g} / \mathrm{mL}$; (R2), $0.19 \mu \mathrm{g} / \mathrm{mL}$; (R3), $0.021 \mu \mathrm{g} / \mathrm{mL}$. Purified substance (A1), $14.81 \mu \mathrm{g} / \mathrm{mL}$; (A2), $1.65 \mu \mathrm{g} / \mathrm{mL}$; (A3), $0.18 \mu \mathrm{g} / \mathrm{mL}$.

from marine fungus A-248 was comparable to antifungal activity of antifungal compound against $P$. oryzae reported in previous studies.

The anti ungal compound was soluble in acetone, ethyl acetate, chloroform, methanol and dimethylsulfoxide, but was insoluble on distillated water. The antifungal substance strongly inhibited the mycelial growth of $P$. oryzae and induced morphological changes of the mycelia. These results were very similar to the antifungal mechanisms of rhizoxin (Kobayashi et al., 1996), the commercial antifungicidal agen. Therefore, the substance purified from A-248 may prove to be valuable as a fungicidal agent of rce blast disease. Further researches are required tc find out the chemical structure of the antifungal compound.

\section{Acknowledgments}

This study was supported from Japan-Korea Industrial Teshnology Co-operation Foundation (JKF). I would like to thank Dr. Shizuri, and Dr. Zhang for their valuable comments on marine microorganism. Thanks also goes to another Marine Biotechnology Institute (MBI) researchers and staff for their kind support and help. Thanks to Ms Kato and other assisting people, who always gave kind help in experiments.

\section{References}

Bae, D.W., Y.S. Kawk, J.T. Lee, D.Y. Son, S.S. Chun and H. K. Kim. 2000. Purification and characterization of a novel antifungal protein from Paenibacillus macerans PM1 antagonistic to rice blast fungus, Pyricularia oryzae. Microbiology and Biotechnology, 10, 805 810.

Bell, A.A. and M.H. Wheeler. 1986. Biosynthesis and functions of fungal melanin. Annu. Rev. Phytopathol., 24, $411 \sim 451$.

Beppu, T. 1993. Morphological abnormalities of eukaryotic cells induced by microbial metabolites as indices to direct nobel physiological activities. Protein, Nucleic Acid and Enzyme, 38, 1639 1646.

Bonman, J.M., G.S. Khush and R.J. Nelson. 1992. Breeding rice for resistance to pests. Annu. Rev. Phytopathol., 30, $507 \sim 528$.

Gunji, S., K. Arima and T. Beppu. 1983. Screening of antifungal antibiotics according to activities inducing morphological abnormalities. Agric. Biol. Chem., 47, 2061 2069.

Inoue, A., S. Tamogami, H. Kato, Y. Nakazato, M. Akiyama, O. Kodama, T. Akatsuka and Y. Hashidoko. 1995. Antifungal melampolides from leaf extracts of Smallanthus sonchifolius. Phytochemistry, 39, 845 -848.

Kim, Y.S., J.K. Son, D.C. Moon and S.D. Kim. 1997. Isolation and structure determination of antifungal antibiotics from Bacillus subtilis YB-70, a powerful biocontrol agent. Kor. J. Appl. Microbiol. Biotechnol., 25, 62 67 .

Kobayashi, H., M. Namikoshi, T. Yoshimoto and T. Yokochi. 1996. A screening method for antimitotic and antifungal substances using conidia of Pyricularia oryzae, modification and application to tropical marine fungi. J. Antibiotics, $49,873 \sim 879$.

Kodama, O., J. Miyakawa, T. Akatsuka and S. Kiyosawa. 1992. Sakuranetin, a flavanone phytoalexin from ultravioletirradiated rice leaves. Phytochemistry, 31, 3807 3809

Lange, L., J. Breinholt, F.W. Rasmussen and R.I. Nielsen. 1993. Microbial fungicides the natural choice. Pestic. Sci., $39,155 \sim 160$.

Manandhar, H.K., H.J. Lyngs Jorgensen, S.B. Mathur and V. Smedegaard-Petersen. 1998a. Suppression of rice blast by preinoculation with avirulent Pyricularia oryzae and the nonrice pathogen, Bipolaris sorokiniana. Phytopathology, $88,735 \sim 739$.

Manandhar, H.K., H.J. Lyngs Jorgensen, V. SmedegaardPetersen and S.B. Mathur. 1998b. Seedborne infection of rice by Pyricularia oryzae and its transmission to seed- 
lings. Plant Disease, 82, 1093 1099.

Padmavati, M., N. Sakthivel, K.V. Thara and A.R. Reddy. 1997. Differential sensitivity of rice pathogens to growth inhibition by flavonoids. Phytochemistry, 46, 499 502.

Richmond, D.V. 1975. Effects of toxicants on the morphology and fine structure of fungi. Adv. Appli. Microbiol., 19, 289 -319.

Sakamoto, K., S. Izumi, M. Miyachi and M. Okuhara. 1995. A new assay method for immunosuppressants with a tacrolims (FK 506)-like mode of action. J. Antibiotics,
$48,727 \sim 729$.

Suzuki, Y., Y. Esumi, H. Hyakutake, Y. Kono and A. Sakurai. 1996. Isolation of 5-( 8 Z-heaptadecenyl)-resorcinol from etiolated rice seedlings as an antifungal agent. Phytochemistry, 41, 1485 1489.

Yeo, W.H., S.K. Kim, S.S. Kim, S.H. Yu and E.K. Park. 1994. Isolation, physico-chemical properties, and biological activity of new thiopeptide antibiotics, kimorexins. J. Microbiol. Biotechnol. 4, 349 353. 